plane is of great interest and if a sufficient number of cases can be found of a similar nature they may lead to a fundamental revision of our present simple notions concerning the scattering of atoms.

In conclusion I wish to take this opportunity to express my thanks to Professor Henry G. Gale for his interest in this work.

1 J. K. Morse, Proc. Nat. Acad. Sci., 14, 37, 1928.

2 J. K. Morse, Ibid., 13, 227-232, 1927.

${ }^{3}$ H. Mark and E. Pohland, Zeit. Krist., 62, 103-109, 1925.

${ }^{4}$ A. W. Hull, Phys. Rev., 10, 661, 1917.

\title{
RELATIONS OF FIELD-CURRENTS TO THERMIONIC-CURRENTS
}

By R. A. Millikan and C. C. Lauritsen

Norman Bridge laboratory of Physics, California Institute of Technology

\section{Communicated December 8, 1927}

In 1925 Millikan and Eyring ${ }^{1}$ first developed experimentally the quantitative laws governing the extraction of electrons from metals by fields alone, i.e., the laws of "field-currents" as they called them. They first proved that the electrons constituting these field-currents are not identical with thermions as had theretofore been assumed. ${ }^{2}$ Their most significant conclusions are stated by them in the following words, which summarize, as well as we can now do, the relations of field-currents to thermioniccurrents, save for an important addition that is here made:

"(e) New proof that the energy of conduction electrons is independent of temperature. The only preceding theoretical treatment of field currents assumes them to be composed of thermions escaping under their own energy of agitation through a boundary weakened by the external field. This conception is, we think, altogether irreconcilable with the foregoing data. Indeed, the entire independence upon temperature of the field currents over a range of $700^{\circ} \mathrm{C}$. constitutes new and striking evidence that 'equipartition' does not hold at all for the bulk of the conduction electrons in tungsten at ordinary temperatures. For when these conduction electrons escape as thermions, the law governing their escape is

$$
i=A T^{n} e^{-b / T}
$$

in which $b$ is the work function of Richardson. The correctness of this thermionic equation is attested by a vast amount of experimental work. Now the effect of an externally applied field of such direction as to pull out electrons should be simply to weaken the effective value of the work function $b$ and leave $i$ in the foregoing equation varying as rapidly with $T$ as it always does in thermionic experiments. The fact, then, that in 
the present experiments $i$ is not at all dependent upon $T$ over a $700^{\circ}$ interval means that the electrons pulled out by the fields here used are not thermions at all. They might become such at high enough temperatures, but at temperatures up to $1000^{\circ} \mathrm{K}$. the electrons here pulled out can have no assistance at all from temperature in getting out, i.e., they do not share in the thermal energy of agitation of the atoms. $* * *$

"The evidence (on this point) derived from the specific heats of metals is inconclusive, because the number of conduction electrons within a metal is entirely unknown. The present evidence, on the other hand, appears to be direct, accurate, and unambiguous for the non-participation of the great bulk of 'field-current electrons' (conduction electrons) in thermal motion. There must, of course, be sufficient participation by a few conduction electrons to account for the Peltier and thermo-electric effects, but this is quite consistent with the quantum theory considerations here employed. *** From the point of view here taken the conduction electrons are simply the lightest of the elements which, at ordinary temperatures, have taken on practically no kinetic energy, i.e., have zero specific heat, and which reach equipartition only at very high temperatures. Those of the conduction electrons which at any temperature have escaped from this zero-energy condition are the thermions. If they carry the total atomic heat of the electrons and if this atomic heat follows the usual quantum law, then their number should increase, at low temperatures, with $T^{3}$. This would be consistent with Eq. (1).

"( $f)$ Relations of field currents and thermionic currents. We have just seen that at low temperatures field currents bear no relation to thermionic currents and that their observed properties lead to the conclusion that the conduction electrons constituting them possess no agitational (temperature) energies at all. But as shown in Table VII at $1100^{\circ} \mathrm{K}$. thermionic currents of measurable magnitude $\left(10^{-12} \mathrm{amp}\right.$.) begin to appear from tungsten and consequently the thermionic equation begins to show evidences of having a role to play in the character of the emission. Thus with increasing potential gradient, the value of the effective work function $b$ is continually reduced and hence the value of the thermionic part of the current (the increase at $1100^{\circ}$ over that at $300^{\circ}$ ) continually rises as is beautifully shown in Table VII so that it makes as large a percentage of the total current when the latter is $10^{-4}$ amperes (potential difference 5000 volts) as when it is $10^{-8}$ amperes (potential difference 2600 volts).

"Quite similarly, as shown in Table VI, strong fields begin at this temperature to bring into evidence the thermionic part of the current when weak fields are unable to do so. In other words, when the temperature is reached at which the number of thermions escaping without external field is at all comparable with the number constituting the field current at what we have called the critical potential gradient, the decrease in $b$ due to 
increasing field pushes up the number of emitted thermions (the electrons coming out in accordance with Eq. (1)) slightly faster than the increase in field pulls out field-electrons. Another way of saying this is that field currents are a less rapidly rising function of the field than are thermionic currents (those following Eq. (1) with $b$ regarded as a function of the applied field $F$ )."

In order to subject to a still further test the relations of field currents to field $F$, one of us (Lauritsen) in repeating in collaboration with the other the foregoing series of experiments tried the plan of plotting the logs of

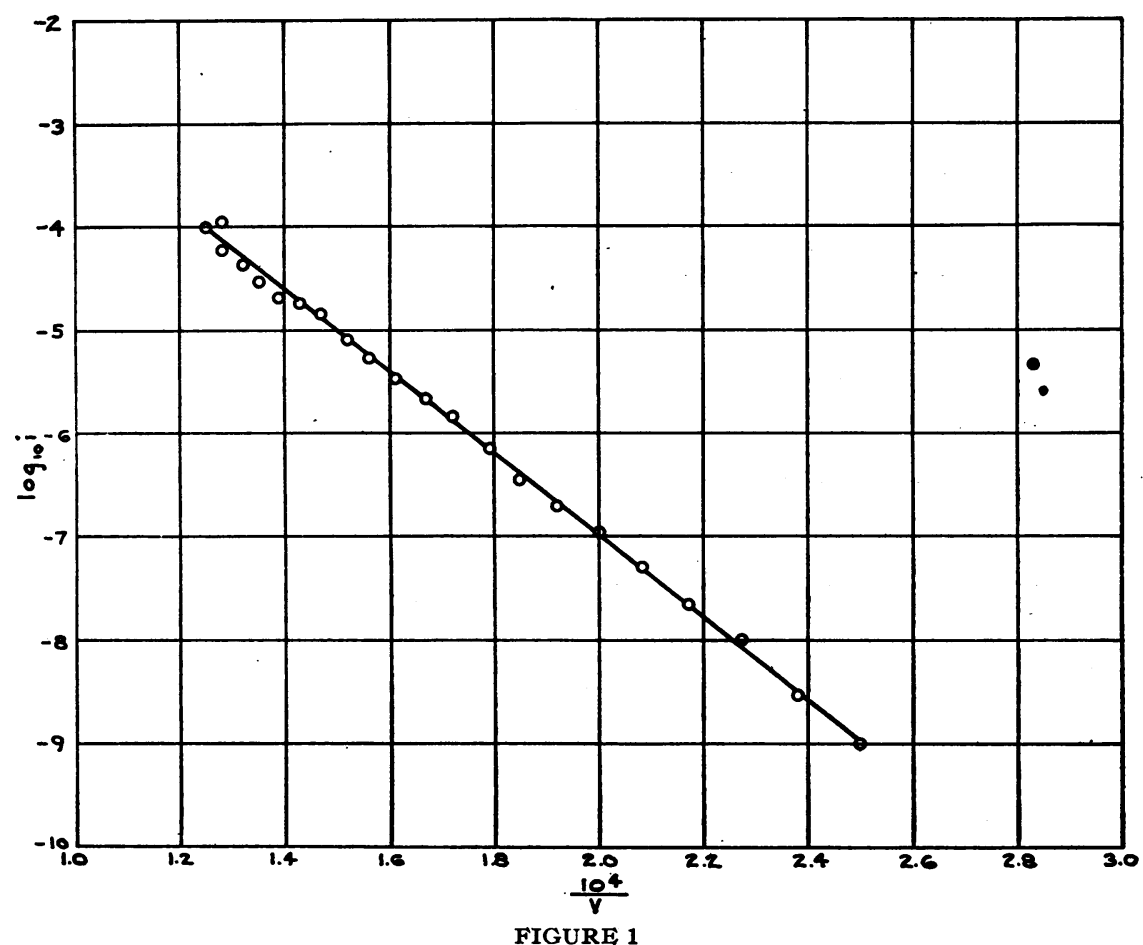

the field currents $(\log i)$ against $1 / F$ instead of against $F^{*}$ or $\sqrt{F}$, as had been done before. The result is shown in figure 1 , which reveals the surprising fact that for these field currents $\log i$ plots as quite as good a straight line against $1 / F$ as is obtained in the thermionic work when $\log i$ is plotted against $1 / T$.

When, next, the Millikan and Eyring data were plotted in this new way. they also yielded in every case a straight line as is illustrated in figure 2 . In this particular case the range of field currents covered will be seen to be

* The abscissæ actually plotted were $10^{4} / V$, since for a given geometrical configuration these are proportional to the $1 / F$. 
from $10^{-11}$ amperes to nearly $10^{-3}$, or an increase of more than fifty million fold.

We have now tested this relation upon the scores' of field current-field relations which have been accumulated in this laboratory over a period of several years, some of them corresponding to currents drawn from fine wires surrounded with cylindrical anodes, some to currents passing between a point and a plane, some with one material and some with another, some with sharp points and some with dull ones, without ever finding a reversible set of field-current readings which did not plot as an excellent straight line.

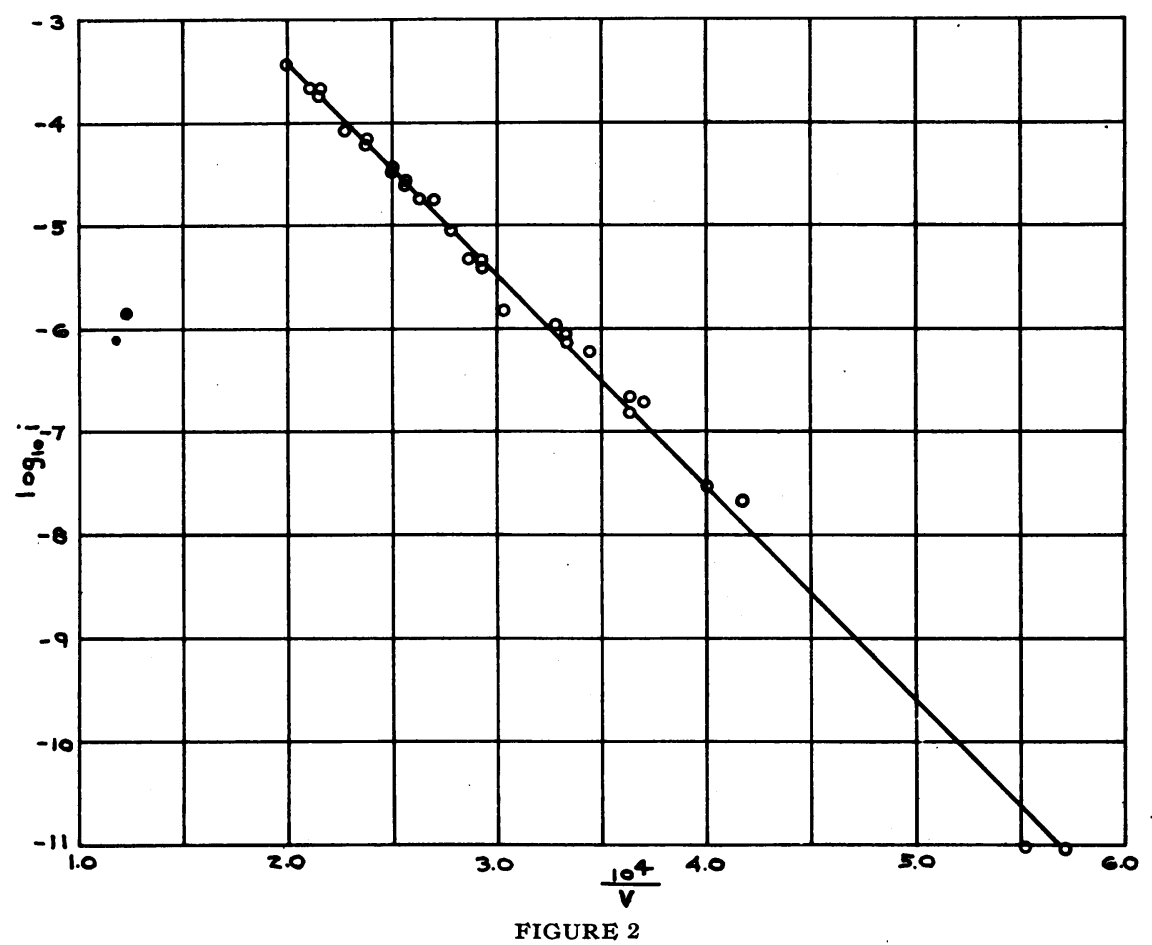

Further, in view of the fact that save at very high temperatures the field currents have been shown to be independent of temperature we are justified in considering that these $\log i-1 / F$ curves woud have precisely the form given above at absolute zero where no thermal effects whatever are possible. This statement brings sharply to the fore the conclusion that the "field-electrons" at ordinary or low temperatures have nothing to do with thermions, i.e., that they must have a static, not a dynamic, interpretation.

The facts presented in $(f)$ above, however, prove definitely that at 
high enough temperatures the fields do extract electrons which share in the energy of thermal agitation (thermions) as well as those which do not. In other words, at high enough temperatures the two effects are not independent.

We are now, therefore, in position to combine the foregoing empirical formula governing field currents, namely,

$$
i=C e^{-b / F}
$$

with the usual formula governing thermionic currents, namely,

$$
i=A e^{-b / T}
$$

and obtain the general formula for the extraction of electrons by fields or by temperature-or by both-in the following form

$$
i=A e^{-\frac{b}{T+c F}} \text {. }
$$

In (2), (3) and (4) $A$ and $C$ may for practical purposes be taken as constants, though for the sake of more exact identification of form with the customary thermionic equation we may, if we wish, write (4) thus

$$
i=A(T+c F)^{2} e^{-\frac{b}{T+c F}} \text {. }
$$

When $F=0$ this obviously reduces to the usual thermionic equation (3) and when $T=0$ (or is small compared to $c F$ ) it reduces to the foregoing field current equation (2) and when $T$ is comparable with $c F$ it yields the combination effects mentioned under $(f)$. The constant $b$ can be taken (or obtained) from thermionic data alone, and then $c$ deduced from the slope of the straight lines shown in figures 1 and 2. The constant $c$, of course, changes with the condition of the surface and we have not yet taken sufficient data to know whether with a given surface one value of $c$ will correspond to all values of $T$.

The new results presented in this paper may be stated thus: The application of an external field is equivalent to increasing the temperature of the electrons within the metal. This is nothing more than what is stated in (2) above, the $T$ of (3) being simply replaceable by the $F$ of (2).

We hope that it is a matter of some importance to have thus obtained a general equation which seems to embrace all the facts of thermioniccurrents and field-currents combined. It may also be that the facts pictured in this equation will be of use in solving the fundamental problems of metallic conduction.

This investigation has been carried out with the aid of funds furnished by the Carnegie Corporation of New York, and administered by the Carnegie Institution of Washington.

${ }^{1}$ Millikan and Eyring, Phys. Rev., 27, 51, 1926.

2 Schottky, Zeit. Physik, 14, 80, 1923. 